\title{
A Platoon Dispersion Model Based on a Truncated Normal Distribution of Speed
}

\author{
Ming Wei, Wenzhou Jin, and Lvou Shen \\ Department of Transportation Engineering, South China University of Technology, \\ Guangzhou 510640, China \\ Correspondence should be addressed to Ming Wei, oscar.weiming@gmail.com
}

Received 24 November 2011; Revised 31 December 2011; Accepted 31 December 2011

Academic Editor: M. F. El-Amin

Copyright ( $) 2012$ Ming Wei et al. This is an open access article distributed under the Creative Commons Attribution License, which permits unrestricted use, distribution, and reproduction in any medium, provided the original work is properly cited.

\begin{abstract}
Understanding platoon dispersion is critical for the coordination of traffic signal control in an urban traffic network. Assuming that platoon speed follows a truncated normal distribution, ranging from minimum speed to maximum speed, this paper develops a piecewise density function that describes platoon dispersion characteristics as the platoon moves from an upstream to a downstream intersection. Based on this density function, the expected number of cars in the platoon that pass the downstream intersection, and the expected number of cars in the platoon that do not pass the downstream point are calculated. To facilitate coordination in a traffic signal control system, dispersion models for the front and the rear of the platoon are also derived. Finally, a numeric computation for the coordination of successive signals is presented to illustrate the validity of the proposed model.
\end{abstract}

\section{Introduction}

At an intersection, lights change from red to green permitting drivers to proceed straight through the intersection. On urban roads these cars will be traveling at different speeds. While moving downstream, the platoon spreads out in a long segment and cars do not uniformly arrive at the next intersection; this is called platoon dispersion. As a platoon moves downstream from an upstream intersection at green phase end time, the cars in the platoon become segmented due to compression and splitting at the downstream intersection's signal lights. It is obvious that using platoon dispersion theory to optimize signal timing plans for traffic signal control could effectively reduce the number of stops and thereby lead to a sharp decline in pollution emissions and fuel consumption.

However, platoon dispersion makes signal coordination more complicated [1-17]. Previous studies on the diffusion of traffic platoons have adopted one of three standard 
approaches: the wave theory of Lighthill and Whitham [1-3], Pacey's diffusion theory [49], and Robertson's recursive platoon dispersion model [10-12]. The first model, also called the LWR model in the literature of traffic flow theory, needs to have an accurate representation of the equilibrium flow-density relationship, which makes it unsuitable for practical applications [13]. The second approach firstly proposes a purely kinematical model to describe the diffusion of traffic platoons by assuming that the speed of traffic follows a normal distribution [4]. Its invalid input parameters (viz. the average speed of vehicles and standard deviation of speed) to calibrate the model make it suitable only for the study of small changes in traffic cycles [6]. The final model uses field data to derive an empirical method for predicting platoon behavior and has been widely used in the well-known TRANSYT software model, because of its simplicity and good explanatory power for understanding the qualitative behavior of road traffic [12]. Both Pacey's and Robertson's models are probabilitybased models with different probability density functions. Seddon [14, 15] concluded in his series of studies on different models of platoon dispersion that there is little difference between the Pacey and Robertson methods with regards to accuracy or efficiency. However, a study by Wang et al. [16] concluded that the recursive model gives good results for short distances, while models using lognormal and normal distributions are better for longer distances.

As mentioned previously, Pacey's model is the most successful combination of theoretical and experimental work on traffic platoons. Most current research based on this model assumes that platoon speed follows a normal distribution, spreading from negative infinity to positive infinity. This does not properly reflect the field situation. Grace and Potts [5] further investigated the density aspect of Pacey's model. Liu and Yang [6-8] proposed a method to correct the vehicle startup time loss of Grace's model. Wang et al. [9] also further refined Pacey's model by considering travel time following a nontransformed normal distribution.

To address the defects of Pacey's model, the authors of this paper propose a more realistic platoon dispersion model, which assumes that the velocity of cars follows a truncated normal distribution, ranging from a minimum speed to a maximum speed [17]. On the basis of the authors' previous research, this paper analyzes platoon dispersion characteristics as the platoon moves down from an upstream intersection at green phase end time. Finally, numeric computation applying the model to signal coordination is presented to confirm the model's validity.

\section{Platoon Dispersion Model}

\subsection{Assumptions of the Speed Density Function}

In Pacey's model, the cars in a platoon are assumed to move with unchanged speeds (i.e., it is ideally treated as the average speed of vehicles measured between adjacent intersections). It is assumed that (a) all the vehicles behind the stop lines uniformly start up after the signal turns from red to green, (b) a car's speed is independent of its position in a platoon, and (c) there is no interaction between cars and a faster car can pass a slower one without hindrance.

A definite value is assigned to the probability that certain cars will have positive speed. Pacey's research has proven that car speeds in a platoon are normally distributed with mean $\mu$ and variance $\sigma^{2}$, which accounts for the spread of platoons. However, the proportion of cars with $v<v_{m}$ and $v>v_{f}$ is zero in reality $\left(v_{m}\right.$ and $v_{f}$ denote minimum speed and maximum 


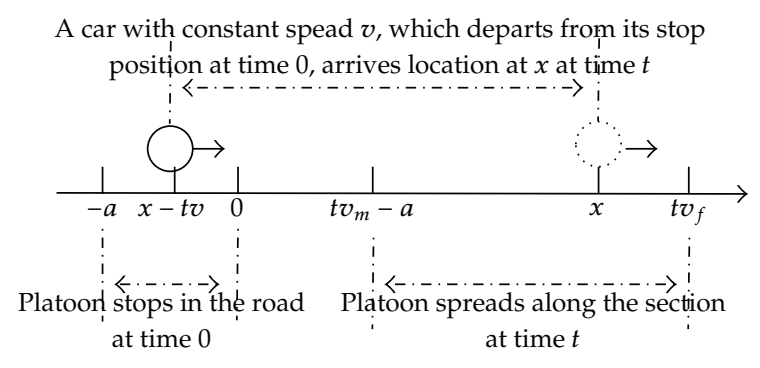

Figure 1: Spreading of platoon.

speed, resp.). Hence, a speed range in a truncated normal distribution extending from $v_{m}$ to $v_{f}$ is a much more reasonable assumption. In this case, the speed density function is as follows:

$$
f^{\prime}(v)= \begin{cases}c \frac{1}{\sqrt{2 \pi} \sigma} e^{-0.5((v-\mu) / \sigma)^{2}}, & v_{m} \leq v \leq v_{f} \\ 0, & \text { others. }\end{cases}
$$

Its calculation formula can be expressed as follows:

(a) for $v<v_{m}: F^{\prime}(v)=\int_{-\infty}^{v_{m}} f^{\prime}(v) d x=0$;

(b) for $v_{m} \leq v \leq v_{f}: F^{\prime}(v)=\int_{-\infty}^{v} f^{\prime}(v) d x=\int_{v_{m}}^{v} f^{\prime}(v) d x=c\left[\int_{-\infty}^{v}(1 /\right.$ $\left.(\sqrt{2 \pi} \sigma)) e^{-0.5((v-\mu) / \sigma)^{2}} d x-\int_{-\infty}^{v_{m}}(1 /(\sqrt{2 \pi} \sigma)) e^{-0.5((v-\mu) / \sigma)^{2}} d x\right]=c\left[F(v)-F\left(v_{m}\right)\right] ;$

(c) for $v>v_{f}: F^{\prime}(v)=\int_{v_{m}}^{v} f^{\prime}(v) d x=\int_{v_{m}}^{v_{f}} f^{\prime}(v) d x=c\left[F\left(v_{f}\right)-F\left(v_{m}\right)\right]=1$.

As the constant $c$ ensures that the accumulated probability of $f^{\prime}(v)$ in the range $\left[v_{m}, v_{f}\right]$ equals 1 , then $1 / c=F\left(v_{m} \leq v \leq v_{f}\right)=\phi\left(v_{f} / \sigma-\mu / \sigma\right)-\phi\left(v_{m} / \sigma-\mu / \sigma\right)$.

\subsection{Development of the Platoon Dispersion Model}

Assuming that the start time of the upstream signal green phase $t$ equals 0 , and the stop bar location $x$ is 0 , then the initial density distribution function $k(x, 0)$ of the queuing vehicles behind the stop bar at time $t=0$ is

$$
k(x, 0)= \begin{cases}0, & x \geq 0 \\ k_{j}, & -a \leq x \leq 0, \\ 0, & x<-a\end{cases}
$$

where $a$ is the queue length and $k_{j}$ is the jam density within the queue length.

This paper studies the movement of a platoon from the beginning of a green phase, until it passes a downstream intersection. As illustrated in Figure 1, the queuing vehicles at the upstream intersection start traveling at constant speed $v$ from their stop position $x-v t \in$ $[-a, 0]$ at the start time of the green phase $t=0$. After time $t$, the number of vehicles having passed the downstream intersection (real or virtual) and the number that have not past the 
downstream intersection are $A(x, t)$ and $B(x, t)$, respectively. The computation formula is derived according to the aforementioned physical definition and presented as follows:

$$
\begin{gathered}
A(x, t)=\int_{x}^{t \cdot v_{f}} k(y, t) d y, \\
B(x, t)=k_{j} a-A(x, t)=\int_{t v_{m}-a}^{x} k(y, t) d y,
\end{gathered}
$$

where $k(y, t)$ is the density of the platoon past $y$ at time $t$. The density distribution function $k(x, t)$, at downstream location $x$ at time $t$, is calculated using the following piecewise function:

$$
k(x, t)=\int_{v_{m}}^{v_{f}} f^{\prime}(v) k(x-v t, 0) d v= \begin{cases}0, & x>t v_{f} \vee x<t v_{m}-a, \\ k_{j} \int_{v_{m}}^{x / t+a / t} f^{\prime}(v) d v, & t v_{m}-a \leq x<t v_{m}, \\ k_{j} \int_{x / t}^{x / t+a / t} f^{\prime}(v) d v, & t v_{m} \leq x \leq t v_{f}-a, \\ k_{j} \int_{x / t}^{v_{f}} f^{\prime}(v) d v, & t v_{f}-a<x \leq t v_{f} .\end{cases}
$$

Let $u=(v-\mu) / \sigma$, and the variation coefficient $\alpha=\sigma / \mu$, as follows:

$$
\int_{v_{1}}^{v_{2}} f^{\prime}(v) d v=c \int_{\left(v_{1}-\mu\right) / \sigma}^{\left(v_{2}-\mu\right) / \sigma} \frac{1}{\sqrt{2 \pi}} e^{-0.5 u^{2}} d u=c \int_{\left(t v_{1} / \mu-t\right) / \alpha t}^{\left(t v_{2} / \mu-t\right) / \alpha t} \frac{1}{\sqrt{2 \pi}} e^{-0.5 u^{2}} d u=\frac{c}{2}[F(z)]_{z_{1}}^{z_{2}}
$$

where $z_{2}=\left(t v_{2} / \mu-t\right) / \sqrt{2} \alpha t, z_{1}=\left(t v_{1} / \mu-t\right) / \sqrt{2} \alpha t, v_{1}$ and $v_{2}$ are constants, and $F(z)=$ $2 \int_{0}^{\sqrt{2} z}(1 / \sqrt{2 \pi}) e^{-0.5 u^{2}} d u=(2 / \sqrt{\pi}) \int_{0}^{z} e^{-u^{2}} d u$ is the standard normal distribution.

Following (2.4) and (2.5), the calculation formula of $k(x, t)$ is as follows:

$$
k(x, t)= \begin{cases}0, & x>t v_{f} \vee x<t v_{m}-a, \\ \frac{c k_{j}}{2}[F(z)]_{\left(t v_{m} / \mu-t\right) / \sqrt{2} \alpha t}^{(x / \mu+a / \mu-t) / \sqrt{2} \alpha t}, & t v_{m}-a \leq x<t v_{m}, \\ \frac{c k_{j}}{2}[F(z)]_{(x / \mu-t) / \sqrt{2} \alpha t}^{(x / \mu+a / \mu-t) / \sqrt{2} \alpha t}, & t v_{m} \leq x \leq t v_{f}-a, \\ \frac{c k_{j}}{2}[F(z)]_{(x / \mu-t) / \sqrt{2} \alpha t}^{\left(t v_{f} / \mu-t\right) / \sqrt{2} \alpha t}, & t v_{f}-a<x \leq t v_{f} .\end{cases}
$$


Let $G(z)=\int F(z) d z=z F(z)+(1 / \sqrt{\pi}) \exp \left(-z^{2}\right)$; then, based on (2.6), the number of vehicles $\int_{x_{1}}^{x_{2}} k(y, t) d y$ distributed along the road segment $\left[x_{1}, x_{2}\right]$ can be calculated as follows:

$$
\begin{aligned}
\int_{x_{1}}^{x_{2}} k(y, t) d y & =\frac{c \cdot k_{j}}{2} \int_{x_{1}}^{x_{2}}\left[F\left(z_{2}(y)\right)-F\left(z_{1}(y)\right)\right] d y \\
& =\frac{c k_{j} \mu \sqrt{2} \alpha t}{2}\left[\int_{z_{2}\left(x_{1}\right)}^{z_{2}\left(x_{2}\right)} F(y) d y-\int_{z_{1}\left(x_{1}\right)}^{z_{1}\left(x_{2}\right)} F(y) d y\right] \\
& =\frac{c k_{j} \mu \sqrt{2} \alpha t}{2}\left([G(z)]_{z_{2}\left(x_{1}\right)}^{z_{2}\left(x_{2}\right)}-[G(z)]_{z_{1}\left(x_{1}\right)}^{z_{1}\left(x_{2}\right)}\right) .
\end{aligned}
$$

Using (2.6) and (2.7), $A(x, t)$ and $B(x, t)$ can be calculated under the following five scenarios:

(a) for $x>t v_{f}: A(x, t)=0$ and $B(x, t)=\int_{t \cdot v_{m}-a}^{t \cdot v_{m}} k(y, t) d y+\int_{t \cdot v_{m}}^{t \cdot v_{f}-a} k(y, t) d y+$ $\int_{t \cdot v_{f}-a}^{t \cdot v_{f}} k(y, t) d y$

(b) for $t v_{f}-a<x \leq t v_{f}: A(x, t)=\int_{x}^{t \cdot v_{f}} k(y, t) d y$ and $B(x, t)=\int_{t \cdot v_{m}-a}^{t \cdot v_{m}} k(y, t) d y+$ $\int_{t \cdot v_{m}}^{t \cdot v_{f}-a} k(y, t) d y+\int_{t \cdot v_{f}-a}^{x} k(y, t) d y ;$

(c) for $t v_{m} \leq x \leq t v_{f}-a: A(x, t)=\int_{x}^{t \cdot v_{f}-a} k(y, t) d y+\int_{t \cdot v_{f}-a}^{t \cdot v_{f}} k(y, t) d y$ and $B(x, t)=$ $\int_{t \cdot v_{m}-a}^{t \cdot v_{m}} k(y, t) d y+\int_{t \cdot v_{m}}^{x} k(y, t) d y$;

(d) for $t v_{m}-a \leq x<t v_{m}: A(x, t)=\int_{t \cdot v_{m}}^{t \cdot v_{f}-a} k(y, t) d y+\int_{x}^{t \cdot v_{m}} k(y, t) d y+\int_{t \cdot v_{f}-a}^{t \cdot v_{f}} k(y, t) d y$ and $B(x, t)=\int_{t \cdot v_{m}-a}^{x} k(y, t) d y$;

(e) for $x<t v_{m}-a: A(x, t)=\int_{t \cdot v_{m}-a}^{t \cdot v_{m}} k(y, t) d y+\int_{t \cdot v_{m}}^{t \cdot v_{f}-a} k(y, t) d y+\int_{t \cdot v_{f}-a}^{t \cdot v_{f}} k(y, t) d y$ and $B(x, t)=0$.

The platoon dispersion model proposed in this paper is described in the previous section. If $x$ is set as the upstream and downstream signal location, then how dispersion characteristics of the queuing vehicles at the upstream intersection influence the downstream green phase start time setup can be quantitatively analyzed, as the platoon moves down from an upstream intersection at green phase end time to downstream intersection. The results can be used to calculate the traffic flow parameters used in signal coordination, such as delay stop and queue length.

\section{The Front and Rear of a Platoon in the Dispersion Model}

In general, only dispersion behavior at the front and rear of the platoon is important in traffic signal coordination. When adjacent intersections are not too far apart, fast cars at the rear of the platoon are unable to get to the front and the slower cars at the front do not have time to filter back to the rear. Hence, the front and rear of the platoons may be treated separately. 


\subsection{Front of the Platoon}

A good design of coordinated lights aims at reducing the number of platoon leaders stopped at the second intersection before its light turns green. The rear does not significantly affect the front, which helps in the mathematical analysis of the behavior of the front of the platoon. Using Pacey's assumptions, the initial density function $k(x, 0)$ of the front of the platoon is defined by

$$
k(x, 0)= \begin{cases}0, & x>0 \\ k_{j}, & x \leq 0\end{cases}
$$

The density of the front of the platoon past $x$ at time $t$, as obtained from (2.4) and (2.5), is

$$
\begin{aligned}
k(x, t) & =c k_{j} \int_{(x / \mu-t) / \alpha t}^{\left(t v_{f} / \mu-t\right) / \alpha t} \frac{1}{\sqrt{2 \pi}} e^{-0.5 y^{2}} d v \\
& =\frac{c k_{j}}{2}\left[F\left(z_{2}=\frac{t v_{f} / \mu-t}{\sqrt{2} \alpha t}\right)-F\left(z_{1}=\frac{x / \mu-t}{\sqrt{2} \alpha t}\right)\right] \\
& =\frac{c k_{j}}{2}[F(z)]_{z_{1}}^{z_{2}} .
\end{aligned}
$$

As mentioned previously, cars at the front travel at a range of speeds $\left[\mu, v_{f}\right]$, leading to the spread of cars along the section $\left[t \mu, t v_{f}\right]$. Hence, the number of cars $A(x, t)$ from the front of the platoon that have passed the downstream location $x$ at time $t$ can be calculated under the following three scenarios:

(a) for $x \leq t \mu: A(x, t)=\left(\left(c k_{j} \mu \sqrt{2} \alpha t\right) / 2\right)\left[z F\left(\left(t v_{f} / \mu-t\right) / \sqrt{2} \alpha t\right)-G(z)\right]_{0}^{\left.\left(t v_{f} / \mu-t\right) / \sqrt{2} \alpha t\right)}$;

(b) for $t \mu<x \leq t v_{f}: A(x, t)=\left(c k_{j} / 2\right) F\left(\left(t v_{f} / \mu-t\right) / \sqrt{2} \alpha t\right)\left(t v_{f}-x\right)-\left(\left(c k_{j} \mu \sqrt{2} \alpha t\right) /\right.$ 2) $[G(z)]_{(x / \mu-t) / \sqrt{2} \alpha t)}^{\left.\left(t v_{f} / \mu-t\right) / \sqrt{2} \alpha t\right)}$

(c) for $x>t v_{f}: A(x, t)=0$.

\subsection{Rear of the Platoon}

Another goal of signal coordination is to reduce the number of stragglers at the rear who miss the green phase. Assuming the upstream signal green time ends at time 0 , the initial density function $k(x, 0)$ of the rear of the platoon, according to Pacey's assumptions, is defined by

$$
k(x, 0)= \begin{cases}0, & x<0 \\ k_{2}, & x \geq 0\end{cases}
$$

where $k_{2} \leq k_{j}$ is the density near the rear of the platoon. 
Applying a similar limit process to (3.2) to calculate the proportion of the rear of platoon that has past $x$ at time $t$, we obtain

$$
\begin{aligned}
k(x, t) & =c k_{2} \int_{\left(t v_{m} / \mu-t\right) / \alpha t}^{(x / \mu-t) / \alpha t} \frac{1}{\sqrt{2 \pi}} e^{-0.5 y^{2}} d v \\
& =\frac{c k_{2}}{2}\left[F\left(z_{2}=\frac{x / \mu-t}{\sqrt{2} \alpha t}\right)-F\left(z_{1}=\frac{t v_{m} / \mu-t}{\sqrt{2} \alpha t}\right)\right]=\frac{c k_{2}}{2}[F(z)]_{z_{1}}^{z_{2}} .
\end{aligned}
$$

The previous calculations show that the speed of vehicles in the rear of the platoon is less than $\mu$, which means that these vehicles are spread out along the section $\left[t v_{m}, t \mu\right]$. Hence, the number of cars $B(x, t)$ from the rear that have not passed the downstream location $x$ at time $t$ can be calculated under the following three scenarios:

(a) for $x \leq t v_{m}: B(x, t)=0$;

(b) for $t v_{m}<x \leq t \mu: B(x, t)=\left(\left(c k_{2} \mu \sqrt{2} \alpha t\right) / 2\right)[G(z)]_{\left(t v_{m} / \mu-t\right) / \sqrt{2} \alpha t}^{(x / \mu-t) / \sqrt{2} \alpha}-\left(c k_{2} / 2\right)(x-$ $\left.t v_{m}\right) F\left(\left(t v_{m} / \mu-t\right) / \sqrt{2} \alpha t\right) ;$

(c) for $x>t \mu: B(x, t)=\left(\left(c k_{2} \mu \sqrt{2} \alpha t\right) / 2\right)\left[-z F\left(\left(t v_{m} / \mu-t\right) / \sqrt{2} \alpha t\right)+G(z)\right]_{\left(t v_{m} / \mu-t\right) / \sqrt{2} \alpha t}^{0}$.

\section{Numerical Calculation}

Form proposed model, $k_{j}$ and $k_{2}$ are not related with diffusion of platoons, which arises only from the differences in speed between vehicles ( $k_{j}$ and $k_{2}$ only decide how many cars are spreading along road). Wei et al.'s study shows that the initial flow is approximately a rectangular pulse with maximum flow $Q=k_{j} \mu=k_{2} \mu$ [17]. Hence, $k(x, t), A(x, t)$, and $B(x, t)$ could be replaced by $k(x, t) / Q, A(x, t) / Q$, and $B(x, t) / Q$ to calibrate the model. Using test data in Grace and Potts's paper [5], upstream intersection $x=0$ and downstream intersection $x=x_{d}$ have the following parameters: $v_{m}=10.1 \mathrm{~m} / \mathrm{s}, v_{f}=33.5 \mathrm{~m} / \mathrm{s}, \mu=13.4 \mathrm{~m} / \mathrm{s}$, and $\sigma=2 \mathrm{~m} / \mathrm{s}$. Generally, if the offset of the downstream intersection is set as $t_{0}=x_{d} / \mu$, then the queuing vehicles at the upstream intersection, travelling at an average speed, can pass downstream intersection at the time.

To verify the validity of the proposed model, this paper compares the difference between the front and rear platoon dispersion characteristics in the proposed model and in Pacey's model. Furthermore, the effect of changing parameter $\alpha$ is examined hereinafter, to quantify how it affects the spreading of the platoon.

\subsection{Platoon Density Distribution Function}

The difference in the density distribution function between proposed model and Pacey's model decides that our model is more realistic than Pacey's model. In order to prove that, $k(x, t) / Q$, which denotes the ratio to the maximum initial flow of density of platoon past $x$ at time $t$, is calculated for both the proposed model and Pacey's model at three time points $t=t_{o} \mu / v_{f}, t_{o},\left(t_{o} \mu+a\right) / v_{m}$ under $x_{d}=45 \mu$, and the results are shown in Figure 2.

The following can be concluded based on Figure 2.

(a) The speed density of both Pacey's model and proposed model follows a normal distribution, which lead to a situation that there are more vehicles traveling 


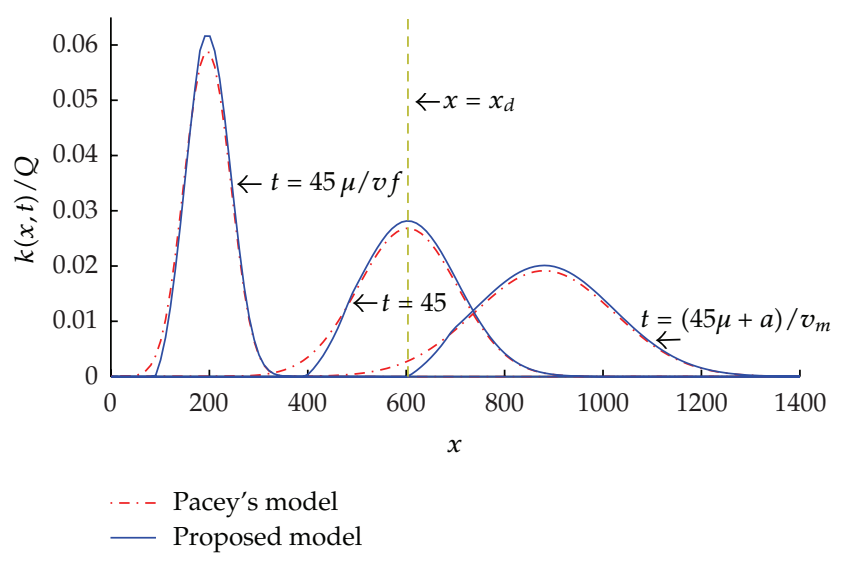

Figure 2: Comparison of the proposed model's and Pacey's density distribution function $k(x, t)$ under $x_{d}=45 \mu$.

around the mean speed, and fewer vehicles at higher or lower speeds. The speed distribution determines the platoon density distribution function. Therefore, the density in the middle of the platoon is higher than that in the front or rear of the platoon. As time passes, the platoon becomes more dispersed along the road, and the hump of the platoon density distribution function becomes less significant.

(b) In the proposed model, the queuing vehicles traveling at different speed $v$ depart from their stop positions at time 0 , and these cars are spreading along the section $x \in$ $\left[t v_{m}-a, t v_{f}\right]$ at time $t$. There are the following three stages: if time is $t \in\left[0, t_{0} \mu / v_{f}\right]$, the front of platoon has not arrived at the downstream intersection $x=x_{d}$, then $t v_{f}<x_{d}$, and $k\left(x_{d}, t\right)=0$; if time is $t \in\left[t_{0} \mu / v_{f},\left(t_{0} \mu+a\right) / v_{m}\right]$, some vehicles are starting to pass the downstream intersection $x=x_{d}$, and $t v_{m}-a \leq x_{d} \leq t v_{f}$ and $k\left(x_{d}, t\right) \neq 0$; if time is $t \in\left[\left(t_{0} \mu+a\right) / v_{m},+\infty\right]$, the rear of the platoon has passed the downstream intersection location $x=x_{d}$, and $t v_{m}-a>x_{d}$ and $k\left(x_{d}, t\right)=0$.

(c) In Pacey's model, the spread of vehicles in the speed range $\left[v_{m}, v_{f}\right]$ during time period $t \in\left[t_{0} \mu / v_{f},\left(t_{0} \mu+a\right) / v_{m}\right]$ is same as proposed model. However, Pacey's model assumes that speed is extending within the range $[-\infty,+\infty]$. It is impossible for a vehicle to have a positive infinity speed to travel to downstream infinity or a negative infinity speed to travel backwards to upstream infinity, which obviously does not match the field observations. Therefore, the application of Pacey's model is limited.

(d) The difference in the speed density between proposed model and Pacey's model decides that there are also more cars in the middle of the platoon and fewer cars in the two tails in the former, compared to the latter.

\subsection{Number of Cars at the Front That Have Passed the Downstream Intersection}

If the green phase of the downstream intersection is started at $t_{0}=x_{d} / \mu$, as cars in the front of the platoon travel at a speed greater than $\mu$, then the downstream signal needs to turn green $t_{h}$ in advance of $t_{0}$ to allow more vehicles to pass during the green phase. That is to say, 
the number of cars stopped at the front of platoon is $A\left(x_{d}, t_{o}-t_{h}\right)$. For $t_{0}=30,60,90,120$ and $t_{h}=0,1,2,10, A\left(x_{d}, t_{o}-t_{h}\right) / Q$, which denotes the ratio to the maximum initial flow of the average number of cars stopped at the front of the platoon, is calculated for both the proposed model and Pacey's model, and the results are presented in Table 1.

Table 1 shows that as the distance between the successive intersections increases, the speed range of the front of the platoon $\left[\mu+a / t_{0}, v_{f}\right]$ increases and $A\left(x_{d}, t_{o}\right)$ also increases. Therefore, a higher preset $t_{h}$ is needed to allow more cars at the front of platoon to pass the downstream intersection. The difference in $A\left(x_{d}, t_{o}-t_{h}\right)$ between the proposed model and Pacey's model is $4.78 \%$, that is, $((c-1) / c) 100 \%$. The difference is a result of the assumption in Pacey's model that the speed spreads from negative infinity to positive infinity, which results in an accumulated probability in the area of $\left[v_{m}, v_{f}\right]$ less than 1.

\subsection{Number of Cars at the Rear That Have Not Passed the Downstream Intersection}

It is assumed that the downstream signal green time ends at time $t_{0}=\left(x_{d}+a\right) / \mu$. According to the platoon dispersion model, the cars at the rear of the platoon are traveling at a speed lower than $\mu$. Therefore, the green phase needs to be postponed to time $t_{t}$ to allow more vehicles at the rear of platoon to pass, which means that the number of vehicles not having passed the downstream signal location is $B\left(x_{d}, t_{t}+t_{0}\right)$. For $t_{0}=30,60,90,120$ and $t_{t}=0,1,2,10$, $B\left(x_{d}, t_{t}+t_{0}\right) / Q$, which denotes the ratio to the maximum initial flow of the average number of cars stopped at the rear of the platoon, is calculated for both the proposed model and Pacey's model, and the results are presented in Table 2.

The data in Table 2 show that as the distance between the successive intersections increases, the speed range of the rear of the platoon, $\left[v_{m}, \mu-a / t_{0},\right]$, increases, and $B\left(x_{d}, t_{o}\right)$ also increases. Therefore, a higher preset $t_{t}$ is needed to allow more cars at the rear of platoon to pass the downstream intersection. As in the analysis of the front of the platoon, the difference in $B\left(x_{d}, t_{t}+t_{0}\right)$ between the proposed model and Pacey's model is $4.78 \%$, that is, $((c-1) / c) 100 \%$.

\subsection{Impact Analysis for Variation Coefficient $\alpha$}

It can be concluded from the previous results that $c$ also determines the deviation of Pacey's model from the real situation. $f^{\prime}(v)$ of $v$ is affected by $\sigma$. As mentioned previously, $\sigma$, relative to $c$, is affected by $\alpha$. Hence, $\alpha$ has some influence on the accumulated probability for speed range $\left[v_{m}, v_{f}\right]$. As $\alpha$ increases, the speed range becomes smaller. This is why the deviation of Pacey's model increases with larger values of $\alpha$.

In addition, $\alpha$ influences the platoon dispersion. A larger $\alpha$ leads to a flatter shape, and a smaller probability around the average speed area; at the same time, the probability for both tails becomes larger. As shown in the derivation process of $k(x, t)$, this is determined by the speed distribution. Thus, the same phenomenon can be seen in the platoon density distribution. To assess the impact of $\alpha$ on $k(x, t), A(x, t) / Q$ of the front and $B(x, t) / Q$ of the rear are calculated separately for $\alpha=0.15$ and $\alpha=0.20$ under $x_{d}=30 \mu$ and the results are shown in Figures 3 and 4. From these figures, we can conclude that as $\alpha$ increases, longer preset and extension times are separately required to allow the cars at the front and the rear of the platoon to pass the downstream intersection. These results are consistent with visual analysis. 


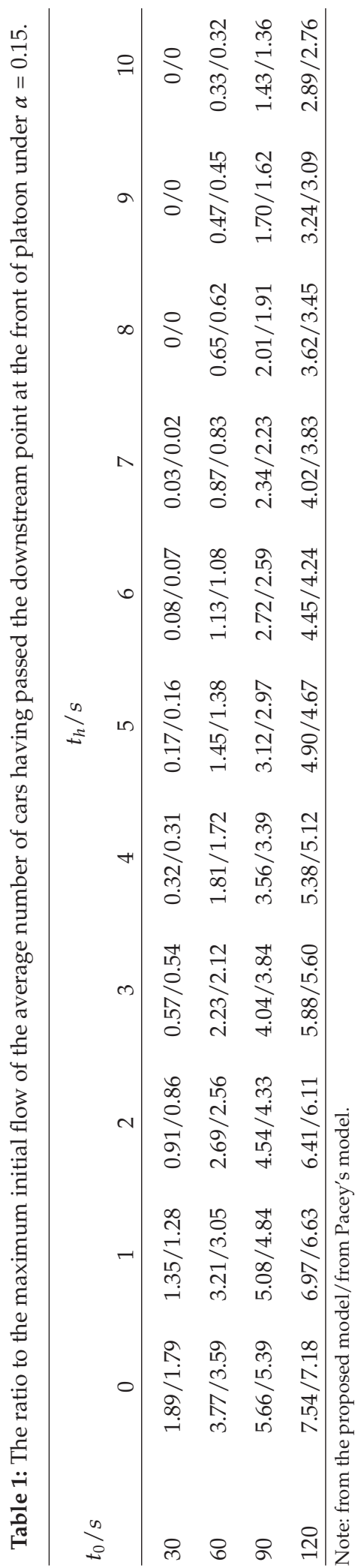


Journal of Applied Mathematics

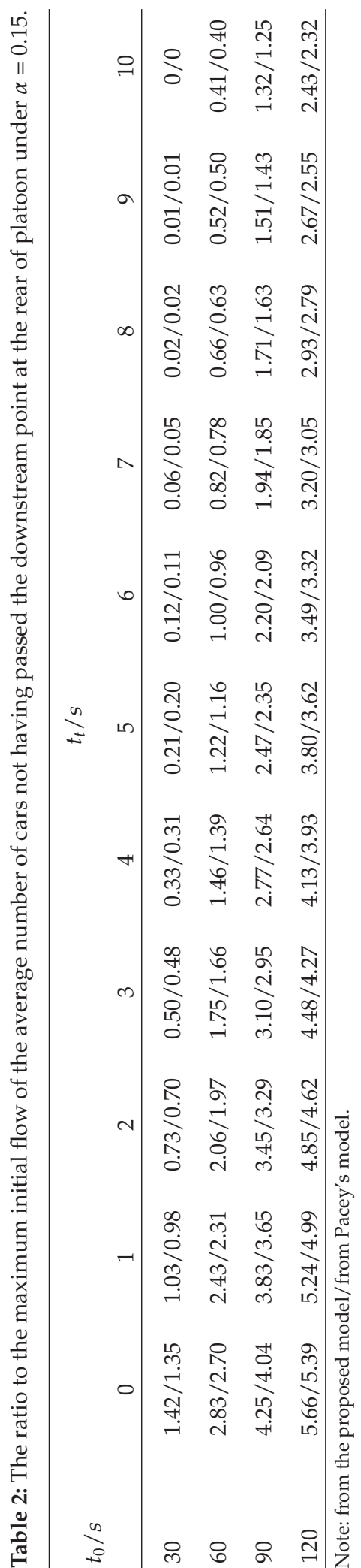




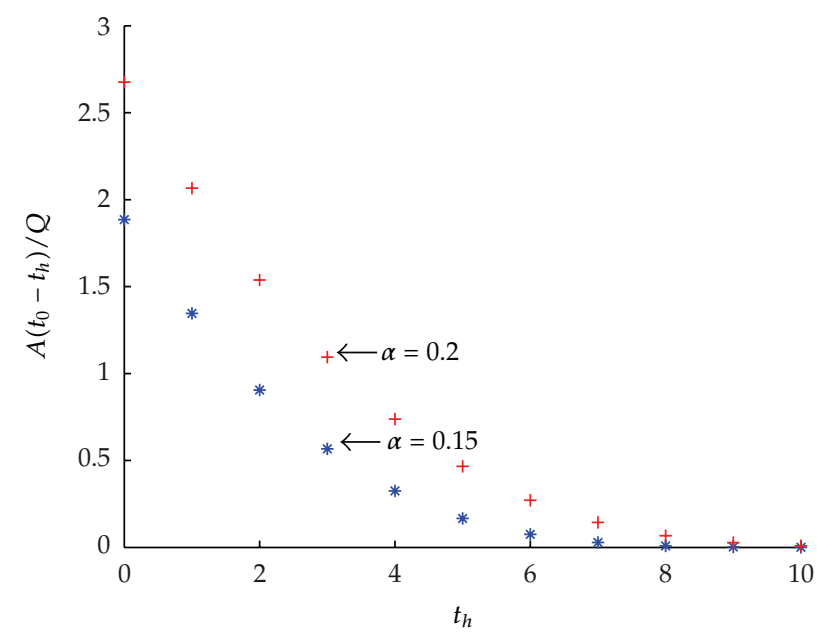

Figure 3: Influence of $\alpha$ on $A(x, t)$ for the front of the platoon.

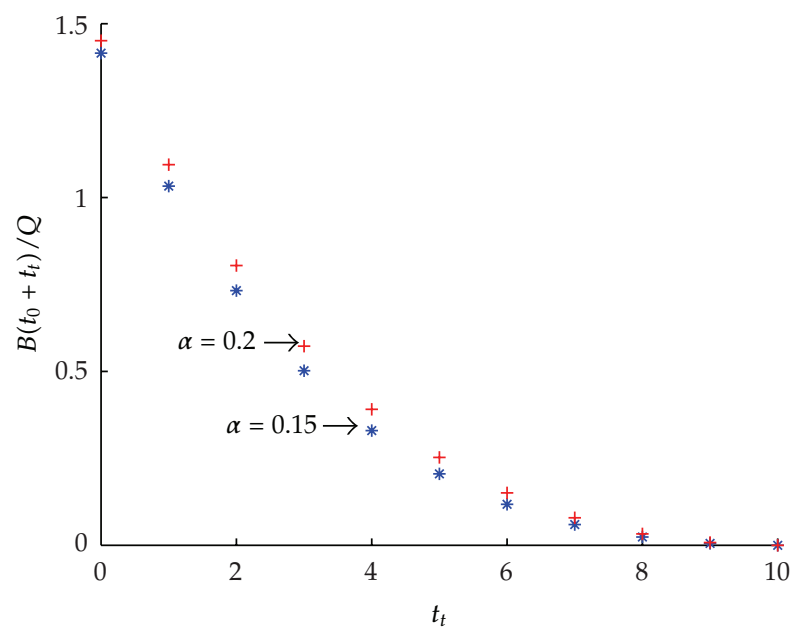

Figure 4: Influence of $\alpha$ on $B(x, t)$ for the rear of the platoon.

\section{Conclusion}

Platoon dispersion is the foundation of coordinating traffic signal control in an urban traffic network. This paper proposes a new platoon dispersion model which assumes that speed density follows a truncated normal distribution. This addresses the main defect of Pacey's model and matches the field situation. To calibrate proposed model, values of four parameters, namely, the average speed of vehicles, the standard deviation of speed, minimum speed, and maximum speed, are quantified. Using test data in Grace and Potts's paper [5] in the numerical example, there are $4.78 \%$ fewer cars travelling in the front and the rear of the platoon between the proposed model and Pacey's model, and we interpret the results for application of coordination of two traffic lights distance $x_{d}$ apart to prove the validity of the proposed model. Future work needs to focus on proposed model with nonsynchronous 
start-up of all vehicles, validating the model using field data, and on simulation program development for the application of the proposed model in the timing of signal coordination.

\section{Acknowledgments}

This work was supported by the High-Technology Research and Development Program of China (863 program) under contract no. 2007AA11Z201 and the National Science Foundation of China under contract no. 61174188. This support is gratefully acknowledged. However, all facts, conclusions, and opinions expressed here are solely the responsibility of the authors. The authors thank the anonymous referee for her/his helpful comments.

\section{References}

[1] M. J. Lighthill and G. B. Whitham, "On kinematic waves:a theory of traffic flow on long crowded roads," Proceedings of the Royal Society of London A, vol. 229, no. 1178, pp. 317-345, 1955.

[2] S. C. Wong and G. C. K. Wong, "An analytical shock-fitting algorithm for LWR kinematic wave model embedded with linear speed-density relationship," Transportation Research B, vol. 36, no. 8, pp. 683706, 2002.

[3] P. Zhang, S. C. Wong, and S. Q. Dai, "A conserved higher-order anisotropic traffic flow model: description of equilibrium and non-equilibrium flows," Transportation Research B, vol. 43, no. 5, pp. 562 574, 2009.

[4] G. M. Pacey, "The progress of a bunch of vehicles released from a traffic signal," Research Note RN/2665/GMP, Road Research Laboraroty, Crowthorne, Berkshire, UK, 1956.

[5] M. J. Grace and R. B. Potts, "A theory of the diffusion of traffic platoons," Operation Research, vol. 12, no. 2, pp. 255-275, 1964.

[6] C. Q. Liu and P. K. Yang, "Diffusion models of traffic platoon on signal-intersection and control of coordinated signals," Journal of Tongji University, vol. 24, no. 6, pp. 636-641, 1996.

[7] C. Q. Liu and P. K. Yang, "Diffusion model of density of traffic platoon and signal coordinated control," China Journal of Highway and Transport, vol. 14, no. 1, pp. 89-91, 2001.

[8] C. Q. Liu and P. K. Yang, "Modification of Grace's density diffusion model and its application," Journal of Highway and Transportation Research and Development, vol. 18, no. 1, pp. 62-64, 2001.

[9] D. H. Wang, F. Li, and X. M. Song, "Modification of Grace's density diffusion model and its application," Journal of Jilin University, vol. 39, no. 4, pp. 891-896, 2009.

[10] D. I. Robertson, "TRANSYT—a traffic network study tool," RRL Report LR 253, Road Research Laboratory, England, UK, 1969.

[11] R. A. Vincent, A. I. Mitchell, and D.I. Robertson, “User Guide to TRANSYT version 8,” RRL Report LR 888, Road Research Laboratory, Crowthorne, Berkshire, UK, 1980.

[12] M. Maher, "A comparison of the use of the cell transmission and platoon dispersion models in TRANSYT 13," Transportation Planning and Technology, vol. 34, no. 1, pp. 71-85, 2011.

[13] P. A. Seddon, "Another look at platoon dispersion: the Kinematic wave theory," Traffic Engineering and Control, vol. 13, no. 8, pp. 332-336, 1971.

[14] P. A. Seddon, "Another look at platoon dispersion: the diffusion theory," Traffic Engineering and Control, vol. 13, no. 9, pp. 388-390, 1972.

[15] P. A. Seddon, "Another look at platoon dispersion: the recurrence relationship," Traffic Engineering and Control, vol. 13, no. 10, pp. 442-444, 1972.

[16] D. Wang, Y. Zhang, and W. Zhitao, "Study of platoon dispersion models," Transportation Research Board Annual Meeting, pp. 3563-3571, 2003.

[17] M. Wei, W. Z. Jin, and L.O. Shen, "A platoon dispersion model considering the speed range limits," Journal of Jilin University. In press. 


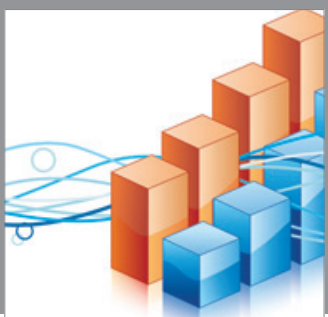

Advances in

Operations Research

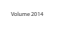

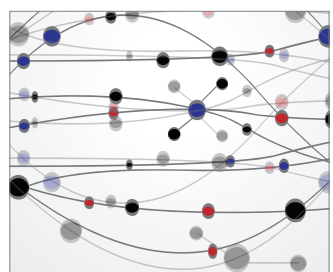

\section{The Scientific} World Journal
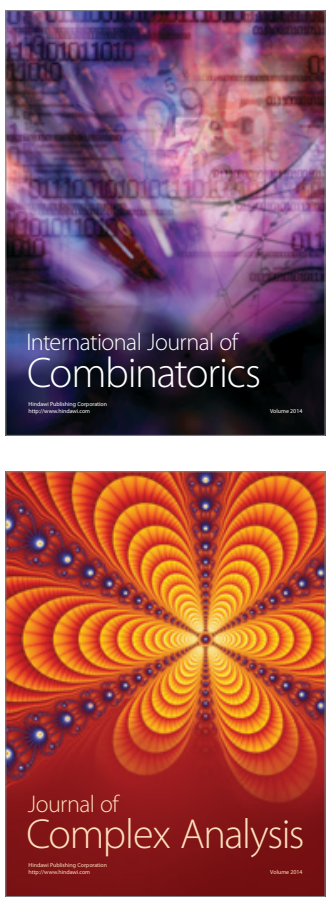

International Journal of

Mathematics and

Mathematical

Sciences
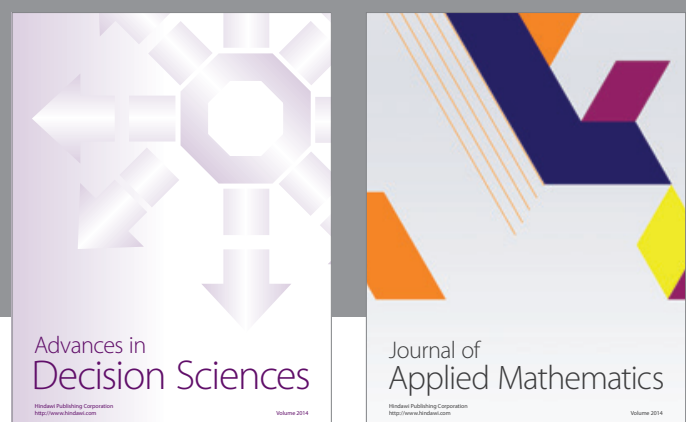

Journal of

Applied Mathematics
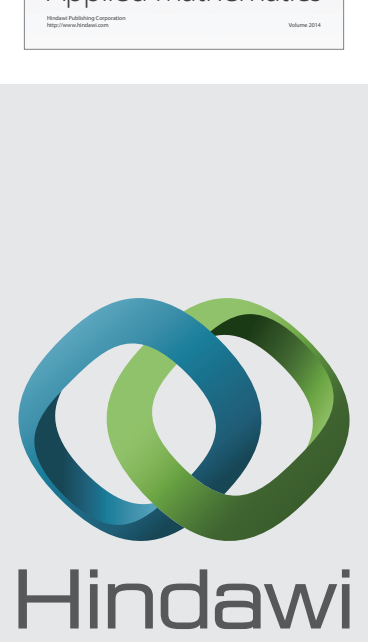

Submit your manuscripts at http://www.hindawi.com
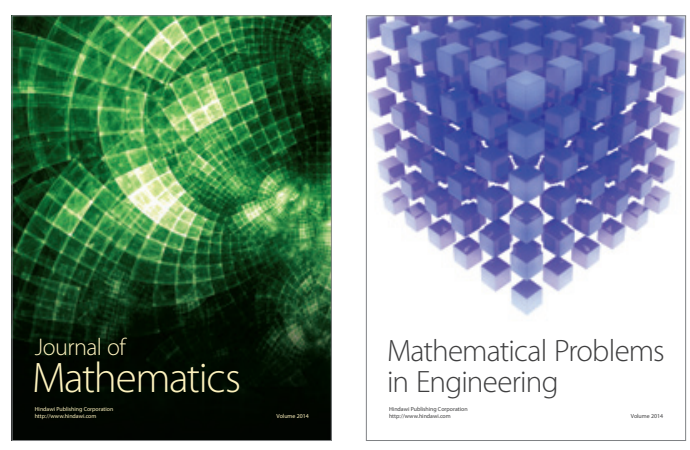

Mathematical Problems in Engineering
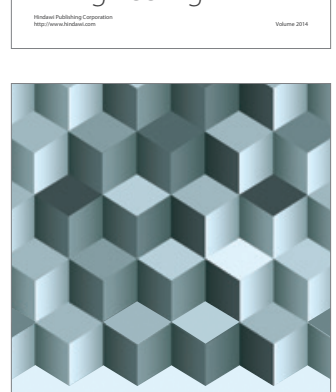

Journal of

Function Spaces
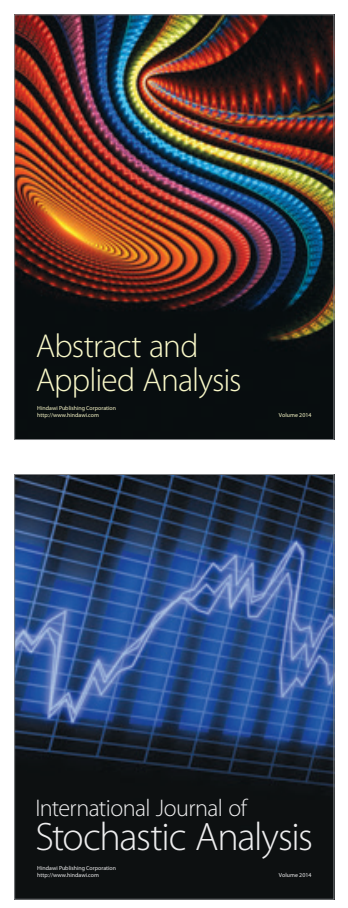

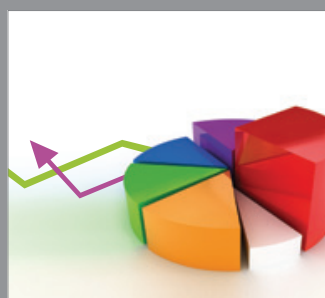

ournal of

Probability and Statistics

Promensencen
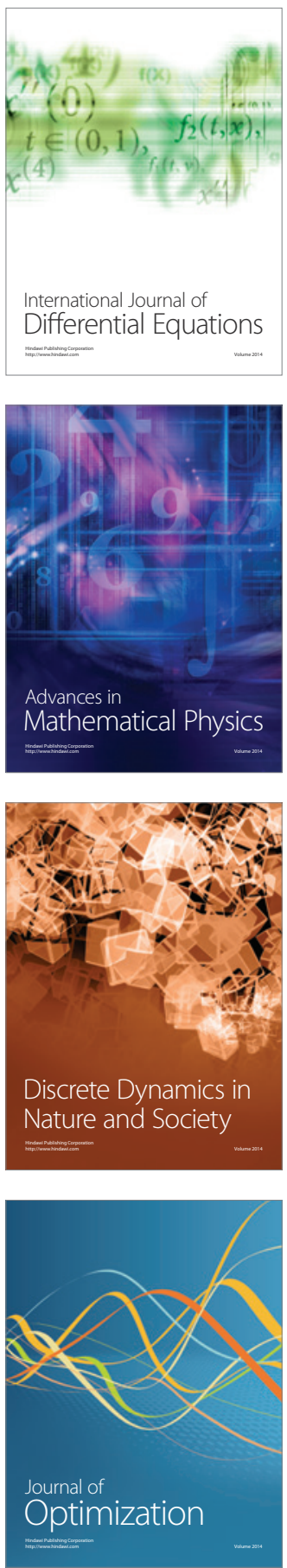